\title{
Late-onset depressive-like behaviors in rats experienced pharmacological stress during early postnatal period
}

\author{
Taku Yamaguchi, Kazuki Baba, Fumi Maeda, Hidenori Matsumoto, Ryo Fukumori, \\ Tsuneyuki Yamamoto
}

Department of Pharmacology, Faculty of Pharmaceutical Sciences, Nagasaki International University, Japan

Objective: Negative life experience such as chronic neglect and maltreatment in the early postnatal period can lead to psychiatric disorders such as depression and anxiety disorder in human. The aim of the present study was to clarify emotional properties in rats repeatedly administered ACTH during the early postnatal period.

Methods: Tetracosactide, the $\mathrm{N}$-terminal 24 amino acids of the naturally occurring ACTH, were administered once a day at dose of $100 \mu \mathrm{g}$ to male rat pups for 5 days on the day 21 after birth (3wACTH). Saline-injected rats were subjected as a littermate control. Emotional properties in 6-, 10- and 25-week-old rats were evaluated by the sucrose preference test and splash test. And we measured the wet weight of adrenal glands in 10- and 25-week-old rats.

Results: Plasma corticosterone in 3wACTH significantly increased in 3 4 weeks old, indicating stress exposure by pharmacological intervention. Ten- and 25-week-old 3wACTH showed the decrease of sucrose consumption in the sucrose preference test, and reduction of time spent grooming after spraying a sucrose solution to the dorsal coat in splash test, but not 6-week-old 3wACTH. These abnormal behaviors in adult 3wACTH indicated the depressive-like/anhedoniclike behaviors, and 3wACTH exhibited late-onset behavioral impairments. Moreover, adrenomegaly observed in 10- and 25-week-old 3wACTH compared with control.

Conclusions: These findings suggest that pharmacological stress (namely ACTH administration) during early postnatal period might produce late-onset depressive-like behaviors in adulthood, which may be due to the abnormal corticosteroid secretion. 